\title{
Bridging the Biophysical-Cultural Divide: The Role of Historical Ecology
}

Throughout the twentieth century, the need to reach scholarly consensus in characterizing the relationship between humans and the environment steadily grew more pressing. Assumptions at the beginning of the century favored a determining role for environment and biology; this was then countered by social scientists, who convincingly demonstrated the enormous role of culture in explaining behavior.

Contemporary research assumes both that humans have altered the environment and that environmental change has shaped the human species and revised human activity. The beginning of the twenty-first century finds humanity with greatly expanded powers to bring about both beneficial and detrimental changes in the global system, but also facing enormous dangers - many, although not all, the result of previous human activity. The quality and quantity of evidence documenting change in the global ecosystem have never been greater but, as understanding of a dynamic Earth system that includes humans increases, discipline-based research frameworks still treat only portions of the system.

The enormous complexity of the human/environment interaction poses an urgent new set of questions: Which human activities impact the global system? How are those impacts manifested? How do global changes threaten human activity? Where are the thresholds beyond which the harm done to human populations and to their environments cannot be repaired? Can human activity that is in accord with the global environment be identified and fostered? In the search for answers, a major challenge is how established scholarly disciplines can collaborate.

In the middle decades of the last century, the complexity involved in answering various aspects of these questions necessitated greater specialization in every field of study. Today, the resultant discipline-based structure of educational and funding institutions has made it immensely more difficult to foster research that untangles circumstances in which both human activity and the global system are in flux. If answers to the new questions are to be found, some basic changes must be made. Disciplines and institutions long accustomed to setting unitary research goals must learn to formulate and carry out collaborative projects, and individual researchers must be rewarded, not punished, when they expand their understanding (if not their expertise) beyond their own training.

What is needed is a flexible framework that integrates biological, physical, and social scientific information with insights from the humanities. Such a framework would focus on spatial and temporal scales that permit the dynamic effects of both human activity and environmental change to be monitored and their links tested. Because values and perceptions motivate human activity, an integrated framework must also include evidence that is difficult to quantify but critical to policy making (Dunlap 1992, 1993; Gore 1994; Kempton et al. 1995; Olsen et al. 1992).

\section{Historical Ecology: Enabling Interdisci- plinary Collaboration}

Several key elements of an integrated framework are now in place. Scientific understanding of the interconnectivity of the atmosphere, hydrosphere, biosphere, and geosphere in the global system is growing (Broecker 1995; Gunn 1991; Lovelock 1989; Root and Schneider 1995; Turner et al. 1989); this parallels earlier research in ecosystematics (Holling 1986; Ellen 1982). Similar developments in social science theory and methods enable comparable evaluation of human activity at several temporal and spatial scales (Balee 1998; Crumley and Marquardt 1987, 1990; Crumley 1993 1994; Gunn 1994a, 1994b; Gunn and Crumley 1991).

This new framework integrates diverse types of evidence, enabling researchers to investigate complex cause-and-effect linkages. Although particular disciplines and individual investigators can make important contributions, development of such an enterprise accelerates when collaborative research projects are made a priority (Chen et al. 1983; Rockwell 1990; Rotberg and Raab 1981). The elements that characterize a new collaborative activity, here termed historical ecology, are appropriately diverse and drawn from several disciplines and intellectual traditions.
Important contributions are the overlapping temporal frameworks that guide both historically-informed social science (e.g., archeology) and the historical sciences such as geology and astronomy. Interpretation, both in history and the sciences, relies on evidence for events and processes that must be set in both immediate and broader temporal and spatial contexts.

By employing temporal and spatial analytic units (such as scale, disturbance, community, landscape, region, niche, boundary, ecotone, etc.) common to several disciplines, diverse fields of study can contribute to a shared language.

Rearchers seeking ways to address time and space simultaneously have found research at the 'landscape' scale particularly useful. Broadly defined as the spatial manifestation of the relations between humans and their environment (Marquardt and Crumley 1987:1), landscape offers a common unit of analysis in several fields (geography, archeology, ecology, geomorphology, architecture, art, regional planning). Landscapes help integrate diverse evidence and allow changes to be traced through time. In conjunction with work in cognition, the study of changes in landscapes (a primary focus in archeology, historical geography, and environmental history) offers practical means of integrating the natural and social sciences and the humanities.

Inasmuch as all research is subject to bias, the more varied the sources of evidence and style of interpretation the more likely we are to effectively characterize a subject. Varied sources of data enable hypotheses to be evaluated with greater independence. Historical ecology challenges all researchers to incorporate both qualitative and quantitative information. The interactive analysis of multiple temporal and spatial scales employs evidence from the prehistoric as well as the recent past, incorporates local and regional spatial data with global data, and serves as a check on assumptions and methods (Marquardt 1992). 


\section{Regional Historical Ecology and Global Change}

The introduction of historically and culturally informed environmental analysis into regional studies offers an important physical basis upon which to practice these new collaborations. Regional studies allow researchers to use previously accumulated data in their models: geology and topography, climate and weather, hydrology, pedology, botany, zoology, and other data are available and, except for the costs of locating them, essentially free. In many regions, historic and contemporary demographic, economic, social, political, and other data are also abundant.

Certain regions of the world are particularly sensitive to environmental changes that affect both human and other living populations. As a laboratory in which previous and current environmental experiments (intentional and unintentional) may be closely analyzed, such regions foster creative thinking about contemporary issues of risk and sustainability. Since the success of mitigation is often determined by how well indigenous cultural practices have been understood, it is important to shape policy that can incorporate local and regional knowledge.

Particularly important to this effort are case studies, which capture both environmental change and human activity over decades, centuries, and even millennia. This broad temporal perspective combined with a manageable spatial unit allows researchers to apportion causation among several factors. Such analyses permit recognition of a "cascade effect" in which one event or circumstance or decision triggers several others in quite different realms that together cause major ecosystemic disruption. Another finding is that the length of time a group has occupied a region is a good rough measure of their ability to use the region's resources wisely.

A period of cultural florescence in one region is sometimes mirrored by eclipse in another. For example, the period AD 500-900 in the Maya lowlands is one of cultural expansion; the same period in Western Europe is characterized by unseasonal weather, famines, pestilence, invasions, and cultural decline, often termed the Dark Ages.

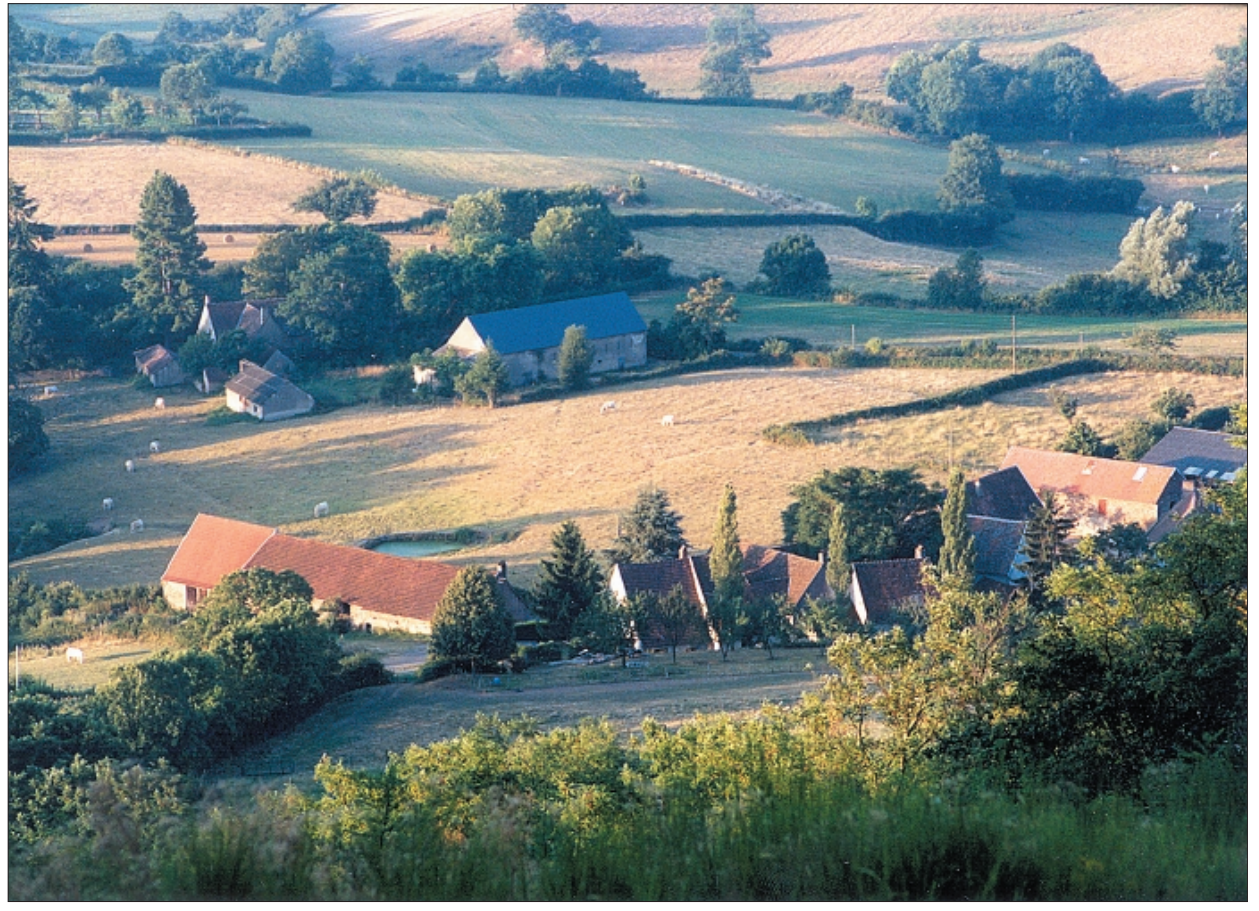

Figure 1: The environmental history of this agricultural landscape in Burgundy, France is being reconstructed by means of palynology, ethnobotany, dendrochronology, historical climatology, fluvial geomorphology, archeology, documentary research and ethnography.

It is just as important to understand the dynamics of a landscape that expresses resilience and adaptation as it is to document the dynamics of past landscape degradation.

This may not be so mysterious once the climatic and marine history of the Atlantic Basin and consequent effects on regions that lie at its margins are better understood. After AD 900 the rainy season was diminished in magnitude and its timing changed, with myriad effects on the Maya environment, economy, and society. In Western Europe a more favorable growing season after AD 900 increased harvests and helped usher in the economic and cultural attainments of the High Middle Ages. These and other examples demonstrate that while many factors can cause economic and social disruption, chief among them is a reduction in environmental diversity coupled with a major change in climate.

Why should all this matter to us today? Human populations' varied responses allow recognition of elements of particular landscapes that successfully maintain species diversity, soil quality and the like. The examination of historic changes in a region's landscape can thus pinpoint useful strategies for long term landscape maintenance. Historical ecology offers an integrated theoretical framework, draws on diverse studies of long- and shorter-term social and environmental change, and allows the construction and comparison of regional ecologial histories, thus joining human and planetary scales. This powerful explanatory framework requires committed interdisciplinary collaboration and a shared vision of linked scales of time and space from local to global. Through it, it can be seen that each of the world's regions and all its peoples can contribute to our understanding of the global system.

\section{Carole l. Crumley}

Department of Anthropology, University of North Carolina, Chapel Hill, NC, USA

crumley@unc.edu

For full references please consult www.pagesigbp.org/products/newsletters/ref2003.html 